\title{
Study of the Spectrum of MRI Findings in Traumatic Knee Joint
}

\author{
Priya Gaddgi Modi', Anmol Modi ${ }^{2}$ \\ ${ }^{1}$ Assistant Professor, Department of Radiology, Dr B R Ambedkar Medical college, Bengaluru, Karnataka, ${ }^{2}$ Fellow in Body imaging, Columbia Asia hospital, \\ Bengaluru, Karnataka.
}

\begin{abstract}
Background: The knee joint has three components, the lateral tibiofemoral, medial tibiofemoral and patellofemoral joints. Four bands of tissue, the anterior and posterior cruciate ligaments, and the medial and lateral collateral ligaments connect the femur and the tibia and provide joint stability. Subjects and Methods: The study was performed during a time period of 12 months. The results of the patients who had undergone both MR and arthroscopy studies were taken for analysis. Results: The most common age group to be involved was between 41-50 years. The following patterns of knee injuries were seen. Most common injury among cruciate ligaments was ACL tear of which complete tears were more common Posterior cruciate ligament tears were less common. Conclusion: Thus, the presence of an anteromedial femoral condyle bone bruise should increase the level of suspicion of a concurrent PLC Knee injury. In addition, we believe that the presence of a posteromedial tibial plateau bone bruise may be a secondary sign of a potential combined PLC injury in the setting of anterior cruciate ligament tear.
\end{abstract}

Keywords: MRI, Traumatic knee joint, Knee injury.

Corresponding Author: Dr. Anmol Modi, Fellow in Body imaging, Columbia Asia hospital, Bengaluru, Karnataka.

Received: June 2019

Accepted: June 2019

\section{Introduction}

The knee is the largest joint in the body. It is a complex 'hinge' joint made up of the lower end of the femur, the upper end of the tibia and the patella, which slides in a groove on the end of the femur. The knee joint has three components, the lateral tibiofemoral, medial tibiofemoral and patellofemoral joints. Four bands of tissue, the anterior and posterior cruciate ligaments, and the medial and lateral collateral ligaments connect the femur and the tibia and provide joint stability. ${ }^{[1]}$

Strong thigh muscles give the knee, strength and mobility. The surfaces where the femur, tibia and patella touch are covered with articular cartilage, a smooth substance that cushions the bones and enables them to glide freely. Semicircular rings of tough fibrous-cartilage tissue called the lateral and medial menisci act as shock absorbers and the bones of the knee are surrounded by a thin, smooth tissue capsule lined by a thin synovial membrane which releases a special fluid that lubricates the knee, reducing friction to nearly zero in a healthy knee. ${ }^{[2]}$

MRI of knee is performed using transmit / receive general purpose extremity surface coil. Quadrature and phase array coils are also available. Increased spatial resolution and decreased signal to noise ratio are significant advantages of these coils.

Imaging is done with full extension in neutral position. A 14 to $16 \mathrm{~cm}$ field of view and a $3-4 \mathrm{~mm}$ slice thickness. MRI is obtained in the Axial, Sagittal and coronal views.

A wide variety of MRI pulse sequences can be performed to produce diagnostic quality images. These include spin echo, fast (turbo) spin-echo, and gradient-echo sequences, which all have been proven suitable for knee imaging. T1 or proton density-weighted sequences are most suitable for visualizing the ligamentous anatomy. T2 or STIR sequences with fat saturation are essential to demonstrate bone marrow edema. Typically, a routine scanning protocol would consist of a combination of one or more of these sequence types performed in the axial, sagittal, and coronal planes using thin sections (maximum $3 \mathrm{~mm}$ with an interslice gap of 0.5 to $1 \mathrm{~mm}$ ). A field-of-view of 12 to $16 \mathrm{~cm}$ depending on patient size is commonly used with a high-resolution matrix of at least 140 steps in the phase-encoding direction. ${ }^{[3]}$

Synovial Membrane appears on both sagittal and coronal images as linear medium signal intensity on T1 weighted and proton density images and as high signal intensity on T2 weighted images. Bursae around the knee joint are not usually seen on MRI unless they are inflamed and fluid filled. ${ }^{[4]}$

ACL is best seen on sagittal, oblique images with slices oriented parallel to the cortex of the lateral femoral condyle. It may appear as a solid band or as three or four separate low signal intensity bands. The tibial attachment is usually better seen than the femoral attachment because of partial volume averaging with lateral femoral condyle. Signal intensity on $\mathrm{T} 1$ weighted and spin density images at the tibial insertion is increased. The anterior border of taut ACL should nearly parallel the roof of the intercondylar notch. Coronal and axial images are useful in confirming the findings made on sagittal images. Coronal images show the 


\section{Modi d Modi; Spectrum of MRI Findings in Troumatic Fnee Joint}

ACL as a curvilinear fan like structure adjacent to the horizontal segment of PCL, near the medial surface of the lateral femoral condyle. Axial images depict ACL as low signal band that is flattened against the medial surface the lateral femoral condyle. All imaging sequences demonstrate fat at the intercondylar notch. ${ }^{[5]}$

Sagittal images best depict the PCL, which appears as a uniformly low signal intensity structure with a nearly horizontal take off at the femoral origin and then an abrupt descent at about 45 degrees to the tibia. This angled portion of the ligament is normally directed towards the femur. The menisco- femoral ligaments of Humphrey and Wrisburg are seen as low signal intensity dots anterior and posterior to the PCL and should not be mistaken for displaced meniscal fragments or an intact PCL in presence of tear.

Medial and Lateral Collateral Ligament is best seen on coronal images where it appears as homogenously low signal intensity structure on all pulse sequences .Moderately increased signal intensities may be seen between superficial and deep fibers and below the superficial fibers at the distal tibial attachment site, where fat is normally interposed.

Oblique Popliteal Ligament (Posterior Oblique Ligament) is best seen on coronal and axial images. A coronal oblique plane along the supero inferior course of the OPL (POL) is also optimal for imaging. ${ }^{[6]}$

The sagittal plane is most important plane in assessing the menisci, with a coronal plane providing supportive rather than new information and the axial plane increasing the accuracy of the sagittal and coronal planes when combined. In general, sagittal images optimally show anterior and posterior horns of the medial and the lateral menisci, coronal images help in evaluating the Meniscal bodies and thin axial sections provide an additional view of menisci and their free edges.

The anterior and the posterior horns of menisci appear as isosceles triangles. The posterior horn of medial meniscus is twice the size of anterior horn. The anterior and posterior horns of lateral meniscus are of same size. The posterior horn of either menisci should never appear smaller than the anterior horn . On both sides, the menisci appear as flat bands. On lateral side, the more central slices take on bowtie configuration because of smaller radius of curvature. ${ }^{[4]}$

Mid portion of the knee produce best images of bodies of both menisci. They appear triangular and slightly larger laterally than medially. The capsular attachment on the medial side is incorporated in the tibial and medial collateral ligament. A small amount of fat may be interposed between the body of the medial meniscus and the capsule. On posterior coronal cross sections, the posterior horns appear as flat bands. On lateral cross sections, the popliteal tendon courses upward and laterally at 45 degrees. More anteriorly, the anterior horn of lateral meniscus appears as a band like structure. The anterior horn of medial meniscus extends more anteriorly than that of lateral meniscus. ${ }^{[6]}$

The medial and lateral menisci, the transverse ligament, and the menisco-femoral ligament appear homogenously dark on all pulse sequences. The vascular and avascular zones cannot be distinguished on MRI. The vascularized zone does not demonstrate enhancement with intravenous gadolinium.

\section{Subjects and Methods}

The study was performed during a time period of 12 months. The results of the patients who had undergone both MR and arthroscopy studies were taken for analysis.

\section{Inclusion Criteria:}

1. Patients with history of pain in the knee with or without swelling where MRI was used as a modality in diagnosing the cause.

2. Patients with clinically suspected tears.

3. Patients with restriction of movement at the knee joint following trivial trauma.

4. Only patients on whom arthroscopy was performed within thirty days of MRI were accepted for the study.

\section{Exclusion Criteria:}

1. Patients with acute traumatic fractures on x-ray

2. Patients diagnosed as having osteochondritis on plain x-ray

3. Patients with cardiac pacemakers and metallic implants were no subjected to MRI.

4. Post operative cases

5. Motion disorder and claustrophobia, if severe may make the examination difficult.

\section{Arthroscopy}

Knee joint is the joint in which arthroscopy have its greatest diagnostic and intraarticular surgical applications. Arthroscopy will be performed under strict aseptic precautions in an operating theatre under regional anesthesia. All the patients were screened with preliminary blood investigations and chest radiographs. The patients were put in supine position with the leg slightly abducted and immobilized. Antero-medial and Antero-lateral portals were made. The arthroscope with 30 degree lens used for the diagnostic and operative procedures.

\section{Results}

\begin{tabular}{l}
\hline Table 1: Age Distribution \\
\begin{tabular}{|l|l|l|}
\hline Age & No. Of Patients & Percentage \\
\hline $11-20$ Years & 06 & $08 \%$ \\
\hline $21-30$ Years & 15 & $20 \%$ \\
\hline $31-40$ Years & 15 & $20 \%$ \\
\hline $41-50$ Years & 21 & $28 \%$ \\
\hline $51-60$ Years & 12 & $16 \%$ \\
\hline $61-70$ Years & 06 & $08 \%$ \\
\hline
\end{tabular}
\end{tabular}

\begin{tabular}{l}
\hline Table 2: Showing clinical symptoms based distribution \\
\begin{tabular}{|l|l|}
\hline Presentation & No. of Cases \\
\hline Instability of Knee Joint & 36 \\
\hline Pain & 27 \\
\hline Swelling & 03 \\
\hline Trivial Trauma & 09 \\
\hline
\end{tabular}
\end{tabular}




\section{Madi of Modi; Spectrum of MRJ Findings in Troumatic Fnee Joint}

Table 3: Showing distribution according to duration of symptoms

\begin{tabular}{|l|l|l|}
\hline Duration of symptoms & No. of patients & \% \\
\hline Upto 1 wcck & 16 & 21.6 \\
\hline 1 week -2 week & 14 & 17.1 \\
\hline 2 week -3 week & 6 & 8.1 \\
\hline 3 week -4 week & 7 & 9.9 \\
\hline 1 month -6 month & 17 & 22.5 \\
\hline$>6$ months & 15 & 20.7 \\
\hline Total & 75 & 100 \\
\hline
\end{tabular}

Table 4: Showing distribution in patients with and without joint effusion

\begin{tabular}{|l|l|l|}
\hline Joint effusion & Number $(\mathbf{n}=\mathbf{7 5})$ & $\mathbf{\%}$ \\
\hline Present & 60 & 80.2 \\
\hline Absent & 15 & 19.8 \\
\hline
\end{tabular}

Table 5: showing distribution in patients with and without ACL tear

\begin{tabular}{|l|l|l|}
\hline ACL tear & Number $(\mathbf{n}=\mathbf{7 5})$ & \% \\
\hline Absent & 35 & 46.7 \\
\hline Present & 40 & 53.3 \\
\hline Partial & 35 & 47.4 \\
\hline Complete & 40 & 52.6 \\
\hline
\end{tabular}

Table 6: showing distribution of patients with and without PCL tear

\begin{tabular}{|l|l|l|}
\hline Present & 51 & 68 \\
\hline Absent & 24 & 32 \\
\hline
\end{tabular}

Table 7: showing distribution of patients with and without MCL tear

\begin{tabular}{|l|l|l|}
\hline MCL tear & Number $(\mathbf{n}=\mathbf{7 5})$ & \% \\
\hline Absent & 74 & 98.7 \\
\hline Present & 1 & 1.33 \\
\hline
\end{tabular}

Table 8: showing distribution of patients with and without

LCL tear

\begin{tabular}{|l|l|l|}
\hline LCL tear & Number $(\mathbf{n}=\mathbf{7 5})$ & $\mathbf{\%}$ \\
\hline Absent & 73 & 97.3 \\
\hline Present & 1 & 2.7 \\
\hline
\end{tabular}

Table 9: showing distribution of patients with and without MM tear

\begin{tabular}{|l|l|l|}
\hline MM tear & Number $(\mathbf{n}=\mathbf{7 5})$ & $\mathbf{\%}$ \\
\hline$\Lambda$ bsent & 28 & 37.4 \\
\hline Present & 47 & 62.6 \\
\hline
\end{tabular}

Table 10: showing distribution of patients with and without

LM tear

\begin{tabular}{|l|l|l|}
\hline LM tear & Number $(\mathbf{n}=\mathbf{7 5})$ & $\mathbf{\%}$ \\
\hline Absent & 45 & 60 \\
\hline Present & 30 & 40 \\
\hline
\end{tabular}

Table 11: Showing distribution of patients with or without osseous/osteochondral lesions

\begin{tabular}{|l|l|l|}
\hline & Number $(\mathbf{n}=\mathbf{7 5})$ & \% \\
\hline Absent & 35 & 46.8 \\
\hline Present & 40 & 53.2 \\
\hline
\end{tabular}

Table 12: showing distribution of combined/multiple injuries

\begin{tabular}{|l|l|l|}
\hline Combined / Multiple injuries & Number $(\mathbf{n}=\mathbf{7 5})$ & $\mathbf{\%}$ \\
\hline Isolated & 28 & 38.09 \\
\hline Two injuries & 30 & 40 \\
\hline Three injuries & 12 & 17.14 \\
\hline Four injuries & 4 & 4.8 \\
\hline
\end{tabular}

Table 13: showing percentage of positive cases on MRI

\begin{tabular}{|l|l|l|}
\hline Structure & No. Of Cases & Percentage \\
\hline $\begin{array}{l}\text { Anterior Cruciate } \\
\text { Ligament(ACL) }\end{array}$ & 40 & $53.3 \%$ \\
\hline $\begin{array}{l}\text { Posterior Cruciate } \\
\text { Ligament(PCL) }\end{array}$ & 24 & $32 \%$ \\
\hline Lateral Meniscus (LM) & 30 & $40 \%$ \\
\hline Medial Meniscus (MM) & 48 & $62.6 \%$ \\
\hline $\begin{array}{l}\text { Medial Collateral } \\
\text { Ligament(MCL) }\end{array}$ & 01 & $1.33 \%$ \\
\hline $\begin{array}{l}\text { Lateral Collateral } \\
\text { Ligament(LCL) }\end{array}$ & 02 & $2.66 \%$ \\
\hline
\end{tabular}

\section{Discussion}

Amreen Abdul Bari et al in the study Evaluation of MRI Versus Arthroscopy in Anterior Cruciate Ligament and Meniscal Injuries in the year 2014 showed the sensitivity, specificity, PPV and NPV was calculated (in \%). For ACL it was $87.87,81.57,80.55,88.57$ for MM 93.54, 87.50, 85.29, 94.59 and for LM 77.77, 81.81, 72.41, 85.71 respectively. MRI is a non-invasive, radiation free and an excellent imaging modality to evaluate ligaments of the knee joint and surrounding soft tissue. The diagnostic yield is increased with appropriate use of sequences and proper analysis of images in all planes. Almost all the ligamentous and meniscal injuries can be diagnosed with high level of confidence. Pathological entities should be carefully differentiated from normal variants, pitfalls and artifacts of imaging. [7]

Rudresh Halawar et al in the study Occurrence of isolated and combined anterior cruciate ligament injuries in traumatic knee-by MRI in 2014 concluded that ACL tear noted in 76 (68.5) patients. Isolated injury noted in $35(46.05 \%)$ and combined injuries in $41(53.94 \%)$ patients. Most common combined injury is ACL with medial menisci tear $(31.7 \%)$ and ACL with lateral menisci tear (7\%).Arthroscopy done in 21 patients. There was no statistically significant difference noted in MRI and arthroscopic findings ( $\tilde{a}=1.02) .{ }^{[8]}$

Adil Ismail Nasir et al 2013 in the study the role of MRI in knee joint injuries concluded that MRI can accurately diagnose the ligament injuries of knee joint,which is an ideal technique in the diagnosis of ligament injuries of knee joint, and should be used as a routine examining method. So MRI affect the diagnosis and management of the knee injuries by decreasing the number of arthroscopic procedures, improving clinician diagnostic certainty, and assisting in management decision.MRI of the knee provides the potential for the rapid, definitive diagnosis with a non invasive examination. ${ }^{[9]}$

Saurav Singla, Nitin Kansal et al 2013 conducted a study sensitivity and specificity of MRI versus Arthroscopy in internal derangement of knee concluded that sensitivity and specificity for medial meniscus were $89.5 \%$ and $85.7 \%$ respectively. NPV and PPV were $90 \%$ and $85 \%$ respectively. For LM, sensitivity, specificity, PPV and NPV were $87.5 \%, 93.8 \%, 77.8 \%$ and $96.8 \%$ respectively. For ACL and PCL, the results of MRI were $88.5 \%, 71.4 \%$, $85.2 \%, \quad 76.9$ and $80 \%, 94.3 \%, \quad 66.7 \%$ and $97.1 \%$ respectively. ${ }^{[10]}$ 


\section{Modi d Modi; Spectrum of MRI Findings in Troumatic Fnee Joint}

S Gupta et al conducted Comparison study Of Clinical Examination, MRI And Arthroscopy In Knee Injuries in 2012 concluded that Diagnostic accuracy of MRI was $66.67 \%$ for medial meniscus and $90 \%$ for lateral meniscus GRADE 1 and 2 meniscal tears have low sensitivity 50\% as compared to GR 3 and 4 with $88 \%$. In the case of ACL tears, diagnostic accuracy for both clinical examination and MR examination came out to be $90 \%$ Conclusion: We can avoid diagnostic arthroscopy in patients with ACL and PCL injuries having equivocal clinical and MRI examination and go on for therapeutic modality. In case of meniscal injuries graded as 1 and 2 on MRI, are rarely seen on arthroscopy hence arthroscopy is not required for these meniscal injuries. $^{[11]}$

Mandelbaum et al 2012 done a systematic approach to establish anatomical and pathoanatomical correlations, as well as the role of MRI in the management of knee injuries. Results indicated that for the medial meniscus MRI demonstrated a $95.7 \%$ sensitivity, $81.8 \%$ specificity, $90 \%$ accuracy, $88.2 \%$ positive predictive value (PPV), and 93.1\% negative predictive value (NPV). Imaging of the lateral meniscus demonstrated a 75\% sensitivity, 95\% specificity, 91\% accuracy, 80\% PPV, and 94\% NPV. MRI of the ACL revealed $100 \%$ sensitivity, specificity, accuracy, positive and negative predictive values. MRI is a noninvasive tool which uses no ionizing radiation and can accurately define and characterize anatomy and pathoanatomy. ${ }^{[12]}$

Elvenes et al 2012 Studied Forty-one knees in 40 patients underwent MRI and arthroscopy. Compared with arthroscopy, the sensitivity, specificity, positive predictive value and negative predictive value for MRI for the medial meniscus were $100 \%, 77 \%, 71 \%$ and $100 \%$, respectively, while the values for the lateral meniscus were $40 \%, 89 \%$, $33 \%$ and $91 \%$, respectively. The overall accuracy for MRI of the medial and lateral menisci combined was $84 \%$. On the basis of the high predictive value of negative MRI, we conclude that MRI is useful to exclude patients from unnecessary arthroscopy. ${ }^{[13]}$

DeepaR. et al 2011 Identified the injury patterns in the pediatric knee both overlap and differ from the adult. Differences between the adult and pediatric populations include an open physis, which serves as a relative point of weakness before physeal fusion; changing mechanics; and differences in ligamentous support. When differences in pathology, disease prevalence, and mechanism of injury are acknowledged, a more accurate interpretation of the MRI findings can be rendered in the pediatric population. In addition, awareness and understanding of normal variants and normal evolution of bone marrow signal may aid in the interpretation of MRI and help avoid unnecessary further workup or intervention. ${ }^{[14]}$

\section{Conclusion}

- Magnetic Resonance Imaging of the knee affected the orthopedists diagnosis, improved clinician diagnostic certainty and reduced the need for arthroscopy. Thus MRI contributed in planning the correct treatment of internal derangement of the knee joint.

- Higher tesla machine for example 1.5T machine might have improved sensitivity and specificity.

\section{References}

1. Aydingoz U, Firat AK, Atay OA, Doral MN. MR Imaging of meniscal bucket handle tear; A Review of signs and their relation to arthroscopic classification.Eur Radiol.2003;13(3);618625

2. Sarah Skinner MRI of Knee Aus Fam Physician 2012 Nov;(11):867-9

3. Edwin HG Oei, Jeroren J Nikken, Antonia CM Verstijen, Abida Z Ginai, MG Myriam Hunik MAGNETIC RENOSANCE IMAGING FOR TRAUMATIC KNEE INJURY . A REVIEW ultrasound ct mri 2007:28:141-157

4. David K B Li, Mark E Adams, Pat McConkey, MRI of ligaments and menisci of the knee. Radiol, Clin North Am 1986; 24(2); 209-227

5. SinghJP,GargL,ShrimaliR,Sethia V Imaging of knee with arthroscopic correlation with twisting injuries Indian journal of radiology imaging 2004; 14: 33-40.

6. Mackenzie R, Dixon AK, Keene GS, Hollingworth W, Lomashritte DJ, Villar RN. Magnetic resonance imaging of the knee: assessment of effectiveness. Clin Radiol.1996; 51(4): 245-250.

7. Amreen Abdul Bari et al Evaluation of MRI Versus Arthroscopy in Anterior Cruciate Ligament and Meniscal Injuries J Clin Diagn Res. 2014 Dec; 8(12): RC14-RC18. Published online 2014 Dec 5. Doi.

8. Rudresh Halawar et al Occurrence of isolated and combined anterior cruciate ligament injuries in traumatic knee-by MRI Medica Innovatic7a, December 2014, Volume 3 - Issue 2

9. Adil Ismail Nasir et al the role of MRI in knee joint injuriesInternational Research Journal of Medical Sciences ISSN 2320 -7353 Vol. 1(5), 1-7, June (2013)

10. Saurav Singla, Nitin Kansal et al sensitivity and specificity of MRI versus Arthroscopy in internal derangement of knee, International Journal of Scientific and Research Publications, Volume 3, Issue 4, April 20131 ISSN 2250-3153

11. S Gupta, R Sharma, A Sachar, Y Saini, N Saini. Comparison Of Clinical Examination, MRI And Arthroscopy In Knee Injuries. The Journal of Orthopedic Surgery. 2012 Volume 19 Number 3.

12. Mandelbaum B R,Finerman GA,Reicher MA,Hartzmans, Bassett LW et al.Magnetic Resonance Imaging as a tool for evaluation of traumatic knee injuries.Anatomical and pathoanatomical correlations.

13. Elvenes J, Jerome CP, Reikeras O,Johansen O. Magnetic Resonance Imaging as a screening procedure to avoid arthroscopy for meniscal tears.

14. DeepaR.Pai,Peter J Strouse MRI of the pediatric knee. Andrew G Geeslin,BS and Robert F Laprade. Osseous Injuries Associated with Acute Grade 3 Isolated and Combined Posterolateral Knee Injuries.

Copyright: (C) the author(s), publisher. Asian Journal of Medical Radiological Research is an Official Publication of "Society for Health Care \& Research Development". It is an open-access article distributed under the terms of the Creative Commons Attribution Non-Commercial License, which permits unrestricted non-commercial use, distribution, and reproduction in any medium, provided the original work is properly cited.

How to cite this article: Modi PG, Modi A. Study of the Spectrum of MRI Findings in Traumatic Knee Joint. Asian J. Med. Radiol. Res. 2019;7(1):79-82.

DOI: dx.doi.org/10.21276/ajmrr.2019.7.1.17

Source of Support: Nil, Conflict of Interest: None declared. 